\title{
Vibration Damping of a New Ionic Liquid under Electric Field Effect
}

\author{
M. M. A. Bicak, H. T. Belek, A. Göksenli \\ Ionic liquids are recently-developed smart materials that are not well known by mechanical engineers. They are of great interest due to their \\ non-volatility, viscosity and extremely high electrical conductivity. Up to now, no reports have appeared on their rheological properties \\ under magnetic or electrical fields. \\ In this work, we study the electro-rheological behaviour of a newly presented ionic liquid (2-hydroxyethylammonium formate). \\ Our experiments show that the ionic liquid is not sensitive to magnetic fields. Nevertheless, resonably high damping ratios (42.8\%) have \\ been attained under relatively low electric fields $\left(0.6 \mathrm{kVcm}^{-1}\right)$.
}

Keywords: damping characteristics, ionic liquid, electrorheological fluid.

\section{Introduction}

Electrorheological (ER) fluids are liquids with flow properties that can be changed by stimulation by an electric field. In particular, changes in dynamic viscosity under an electric field have found many applications in various devices such as clutches, brakes, active engine mounts and shock absorbers $[1,2]$. The rheological properties of the oil dispersions of stimulus responsive particles have been well documented. The key principle of an ER fluid is the dispersion of electro-conducting particles in a non-conducting liquid medium.

We considered that it woud be interesting to investigate the rheological properties of an ionic liquid. In this work, we study the rheology of a new ionic liquid in electric and magnetic fields. To the best, of our knowledge, no reports have appeared in the literature dealing with the rheology of ionic liquids.

Ionic liquids are salts which are fluids at around room temperature. In recent years they have attrached increasing interest. Only in 2001-2002 more than five hundred papers were published. Ionic liquids show unusual physical properties such as high ionic conductivity and powerful solution efficiency for various organic and inorganic substrates. Two types of ionic liquids, imidazolinium salts $[3,4,5]$ and trialkylammonium salts [6] have been studied extensively by chemists.

More recently a new type of ionic liquid, 2-hydroxyethylammonium formate was discovered at the chemistry department of our university. According to their report [7], it is obtained simply by mixing ethanolamine and formic acid, both of which are commercially available. The freezing temperature of this salt is $-82{ }^{\circ} \mathrm{C}$, which is the lowest freezing temperature among ionic liquids.

This work deals with measurements of the vibration damping effect of the ionic liquid by the logarithmic degrement approach under magnetic and electric fields. The variation of the damping coefficient is derived and the results are discussed. Our preliminary experiments show that the new ionic liquid is not stimulated by magnetic fields. However, a relatively high vibration damping effect was detected even under low electricfields, compared with the effects discussed in previous reports.

\section{Mechanism of the electro-rheological effect}

The electrorheological effect refers to the sudden and reversible change in the flow characteristics by means of an electric field. An abtract change in the molecular orientation of the structure from an initially random distribution to a more ordered structure takes place. Furusho classified ER fluids into two-phase (particle-type) and one-phase (homogeneous-type) systems in terms of their characteristics [8].

Conventional ER fluids, for example suspensions of polarizable solid particles and insulation oil, demonstrate an orientational change in response to an external electric field because of the induced aggregation of the particles. The main disadvantage of two-phase ER fluids is that their characteristics change greatly with the shape and dimensions of the particles [9].

Homogeneous-type ER fluids have been developed by using a solution of low-molecule liquid crystals or macromolecular liquid crystals $[10,11]$. Up to now, the ER effects of electron conducting dispersed particles have been studied. Here we present the rheological effect of an ionic liquid in which electrical charges are created by ion migration rather than by electron movements.

The ER effect is strongly dependent on polarization rate, permittivity, the dielectric loss factor and conductivity [12]. It is important to note that the structure of the carrier fluid may break down under high electrical fields, when highly conducting substrates are used. Because of this fact, it is not possible to obtain high damping forces. In this case, the dampings received are substantially lower than those produced by magnetorheological fluids. Zhao et al. demonstrated that the ER effect produced by conducting and dielectric systems are completely different in nature [13]. For instance, network systems are formed rather than linearly aligned chain systems. In fact, the conductivity effect dominates in low frequency AC fields, and permittivity dominates at high frequencies. In other words low frequency AC fields must be applied to conductive ER fluids in order to achieve a better ER effect. In this study, we have intestigated the rheological properties of an ionic liquid under DC electric fields. 


\section{Physical properties of the ionic liquid}

Recently a new ionic liquid (2-hydroxyethylammonium formate), with an extremely low melting temperature $\left(-82{ }^{\circ} \mathrm{C}\right)$, has been reported [7]. This ionic liquid shows reasonably high room temperature ionic conductivity $\left(3.3 \mathrm{mScm}^{-1}\right)$ and heat stability up to $150{ }^{\circ} \mathrm{C}$. Other physical properties are given in Table 1 .

Table 1: Some physical characteristics of the ionic liquid*

\begin{tabular}{|c|c|}
\hline Appearance & Viscous clear liquid \\
\hline Density & $1.204 \mathrm{gcm}^{-3}$ \\
\hline Refractive index & $\mathrm{nD}=1.4772\left(\right.$ at $\left.25^{\circ} \mathrm{C}\right)$ \\
\hline Viscosity & $\eta=105 \mathrm{cP}\left(\right.$ at $\left.25^{\circ} \mathrm{C}\right)$ \\
\hline Conductivity & $\sigma=3.3 \mathrm{mScm}^{-1}\left(\right.$ at $\left.25^{\circ} \mathrm{C}\right)$ \\
\hline Decomposition Temperature & Approx. $150^{\circ} \mathrm{C}\left(\right.$ by $\left.\mathrm{TGA}^{\otimes}\right)$ \\
\hline Vapor pressure & $\begin{array}{c}2.2 \times 10^{-2} \text { Torr } \\
(\text { air saturation method) }\end{array}$ \\
\hline Melting point & $\mathrm{Mp}$ (freezing point): $-82^{\circ} \mathrm{C}$ \\
\hline
\end{tabular}

(*adapted from ref. [7], ${ }^{\otimes}$ Thermo gravimetric Analysis)

The room temperature conductivity of the ionic liquid is $3.3 \mathrm{mScm}-1$, which is in the order of metallic conductivities.
The ionic conductivity increases exponentially with temperature, and reaches $40 \mathrm{mScm}^{-1}$ at $92{ }^{\circ} \mathrm{C}$ (Fig. 1). This effect can be attributed to fast ion mobilities at elevated temperatures.

The AC conductivity - frequency plot of the ionic liquid (Fig. 2) shows a sharp increase in the $(0.1-10) \mathrm{Hz}$ range. A plateau appears between $10 \mathrm{~Hz}-10 \mathrm{MHz}$, in which the conductivity is around $68 \mathrm{mS} \mathrm{cm}^{-1}$ at room temperature.

The viscosity of the ionic liquid decreases with temperature. For instance, $105 \mathrm{cP}$ of room-temperature viscosity reduces to $15 \mathrm{cP}$ at $70{ }^{\circ} \mathrm{C}$. Processing of the temperature dependent viscosity data shows an Arhenius type of relationship from which the following correlation is obtained:

$$
\log \eta=-5.265+\frac{1919.5}{T},
$$

where $\eta$ denotes viscosity in terms of $\mathrm{cP}$, and $T$ is temperature $(\mathrm{K})$.

\section{Experimental}

We designed a home-made experimental setup shown in Fig. 3, for damping measurements under an electric field effect. The same setup can also be used under a magnetic field by replacing the electric field with a magnetic coil.

The measurements were carried out under $(0-0.6) \mathrm{kV} \mathrm{cm}^{-1}$ of a DC electric field. For this purpose the damper reservoir was filled with about $25 \mathrm{ml}$ ionic liquid. The electric field was created by two parallel plates using a controllable power supply. Meanwhile, the shaker was stimulated by amplified signals. The oscillations generated by the shaker

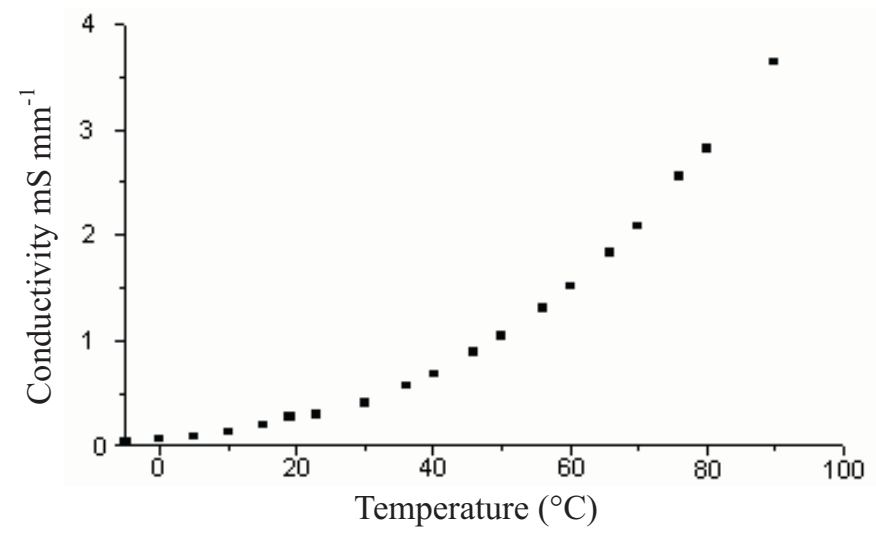

Fig. 1: Effect of temperature on conductivity

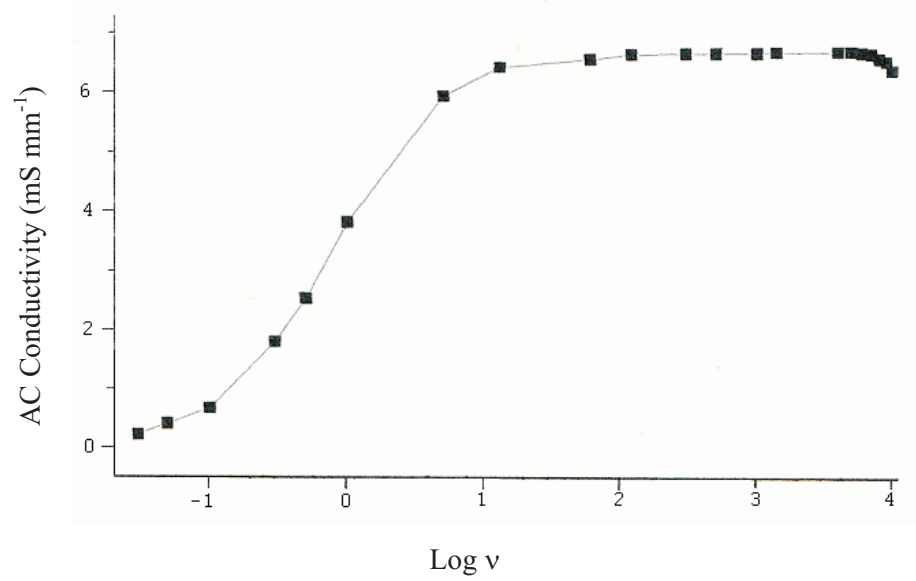

Fig. 2: AC conductivity - frequency plot 


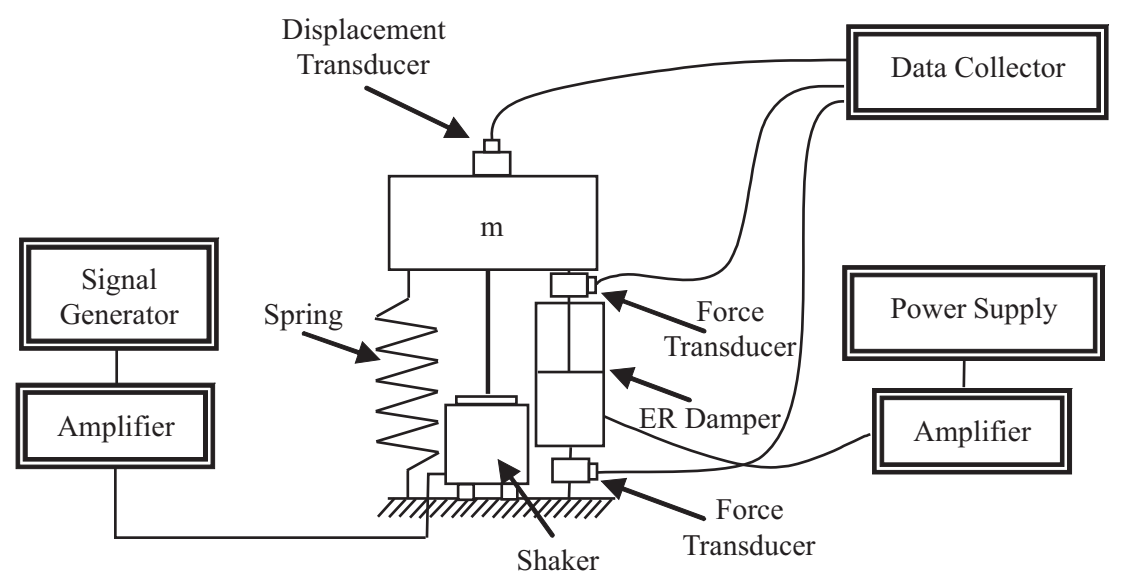

Fig. 3: Schematic diagram of the testing unit

were collected and the output responses were collected by a data collector (Bruel \& Kjaer). The displacements-time plots are illustrated in Fig. 4., and the logarithmic degrement of the oscillations was calculated by tracing the maxima of the displacement signals using the following formula

$$
\delta=\frac{1}{r} \ln \frac{A_{i}}{A_{i+1}}, \zeta=\frac{1}{\sqrt{1+(2 \pi / \delta)^{2}}},
$$

where $A_{i}$ is the first significant amplitude, and $A_{i+r}$ is amplitude after $r$ cycles.
The damping forces were recorded simultaneously by means of the force transducers. These experiments were carried out under various electric fields. The data collected is pictured as a function of the electric field, as shown in Fig. 5.

\subsection{Measurements under a magnetic field}

The experiments described above were repeated under constant and sinusoidal magnetic fields supplied from an electromagnetic coil with an inner diameter of $10 \mathrm{~cm}$. Varia-

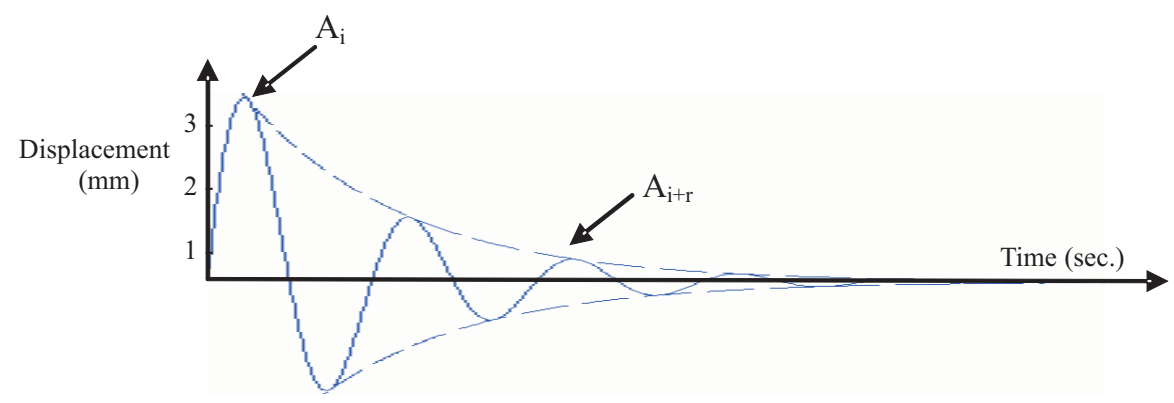

Fig. 4: Impulse response of the oscillator

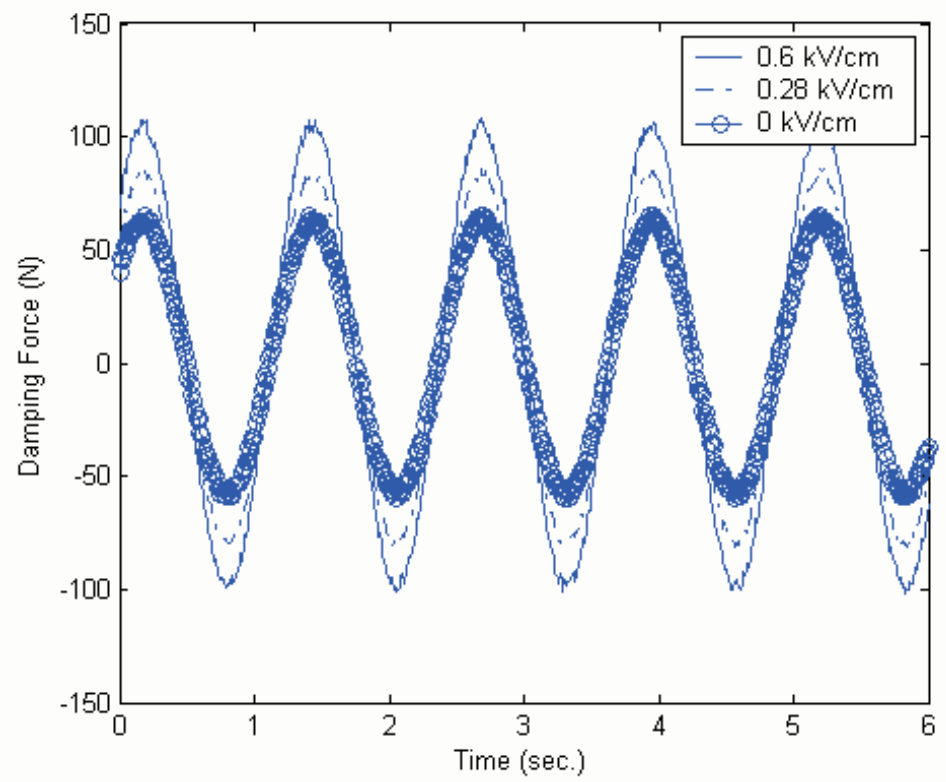

Fig. 5: Damping force - time relationship under an electrical field 
tion of the magnetic field (0-1) Tesla did not give any significant change in response relative to blank experiments.

\section{Results and discussion}

Although we are not able to apply high electric fields, we have observed reasonable rises in damping ratios as high as $42.8 \%$ in moderate electrical fields up to $0.6 \mathrm{kV} \mathrm{cm}^{-1}$ (Fig. 6). This seems to be due to the quick orientation of the dipoles of the ionic liquids under an electric field. This result is especially significant because such high damping can only be achieved under high electric fields (i.e. $40 \mathrm{kV} \mathrm{cm}^{-1}$ ), as described many times in the literature [13]. Since the viscosity of the liquid is inversely proportional to the temperature, high damping performances are expected at lower temperatures.

However, the conductivity of the ionic liquid increases with temperature. In other words, the effects of viscosity and conductivity, in our case, are contraversal in the damping effect. Nevertheless, the high solubility of of the liquid compensates the disadvantage of the temperatures. For instance, the addition of ammonium chloride $\left(\mathrm{NH}_{4} \mathrm{Cl}\right)$ increases both the conductivity and the viscosity at the same time.

It is important to note that there is a great difference between the present system and the reported data obtained from electroconducting particle dispersions. In the present case, electric conduction is provided by ion migration rather than by electron conduction.

In our case, $3.3 \mathrm{mScm}^{-1}$ of conductivity at room temperature is comparable with the conductivities of conducting metals. In other words, the conductivity of the ionic liquid is about $10^{6}$ times that of particle dispersions reported so far.

Better damping effects can be attained by dissolving dissociable salts in the ionic liquid at low temperatures. The advantage of the liquid presented here over reported systems is that it can be stimulated by low electric field strengths. The non-volatility of the liquid is another advantage.

We have also studied the damping response under a magnetic field in the (0-1) T range supplied by a magnetic coil. However, no significant response was detected, as expected.

\section{Conclusion}

Ionic liquids are ion conducting viscous liquids which provide reasonable dampings under relatively low electrical fields. The new ionic liquid without any added ingredient does not show any magnetorheological effect. However, due to its powerful solvating effect, the homogeneous or semi-homogeneous magnetic particle dispersions are of interest. Further studies are under consideration.

\section{Acknowledgments}

We are grateful to Prof. N. Bicak for his valuable help in donating the ionic liquid.

\section{References}

[1] Carlson, J. D., Catanzarite, D. M., St. Clair, K. A.: Electrorheological Fluids, Magnetorheological Suspensions and Associated Technology (ed. W. A. Bullough). Singapore: World Scientific, 1996, p. 20.

[2] Wu, X. M., Wong, J. Y., Sturk, M., Russell, D. L.: Electrorheological Fluids: Mechanisms, Properties, Technology and Applications (ed. R. Tao and G. D. Roy). Singapore: World Scientific, 1994, p. 538.

[3] Watanabe, M., Yamada, S. I., Ogata, N.: Electrochim. Acta, Vol. 40 (1995), p. 2285-2288.

[4] Fuller, J., Breda, A. C., Carlin, R. T.: J. Electrochem. Soc. Vol. 144 (1997), No. 4, L67-L70.

[5] Noda, A., Watanabe, M.: Electrochim. Acta, 45 (2000), p. 1265-1270.

[6] Forsyth, M., Sun, J., MacFarlane, D. R.: Electrochim. Acta, Vol. 45 (2000), p. 1249-1254.

[7] Bicak, N.: "A New Ionic Liquid: 2-Hydroxy Ethyl Ammonium Formate." J. Molecular Liquids, (in Press)

[8] Furusho, J.: "Control of Mechatronics Systems Using Electrorheological Fluids." Journal of the Society of Instrument and Control Engineers, Vol. 34 (1995), p. 687-691.

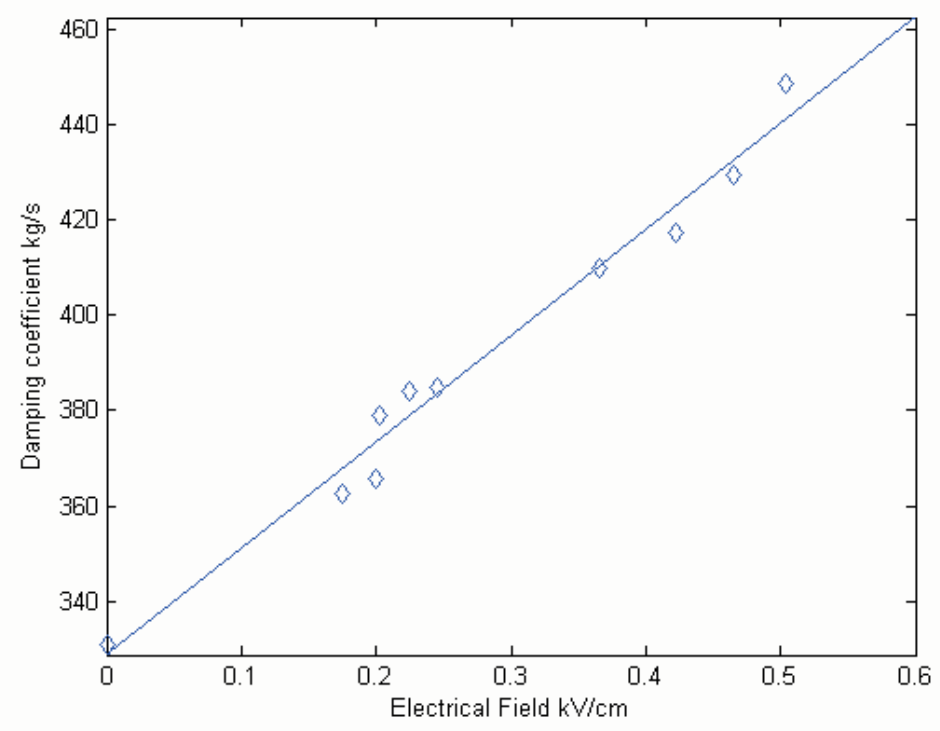

Fig. 6: Effect of an electrical field on damping coefficients 
[9] Johson, A. R., Makin, J., Bollough, W. A.: Advances in Electrorhological Fluids (ed. M. A. Kohudic). Technomic Publ., 1994.

[10] Inoue, A., Maniwa, S.: "Electrorheological Effect of Liquid Crystalline Polymers." Journal of Application Polymer Science, Vol. 55 (1995), p. 113-118.

[11] Inoue, A.: "Trends of Homogeneous ER Fluid Development." Journal of the Society of Instrument and Control Engineers, Vol. 34 (1995), p. 698-701.

[12] Akhavan, J., Slack, K., Wise, V., Block, H.: "Coating of Polyaniline with an Insulating Polymer to Improve the Power Efficiency of Electrorheological Fluids." Proceedings of the $6^{\text {th }}$ International Conference on Electrorheological Fluids, Magnetorheological Suspensions and Their Applications, eds. M. Nakano, K. Koyama, 1997, p. 322.

[13] Zhao, X. P., Chen, J., Shen, Y. X., Lu, K. Q.: "Properties of Conductive Electrorheological Systems.” Proceedings of the $6^{\text {th }}$ International Conference on Electrorheo- logical Fluids, Magnetorheological Suspensions and Their Applications, eds. M. Nakano, K. Koyama, 1997, p. 302.

M. M. Altug Bicak

Tel.: + 90-212-293 13 00/2510

Fax.: +90-212-2450795

e-mail: bicakme@itu.edu.tr

H. Temel Belek

Tel.: +90-212-2931300/2577

e-mail: belek@itu.edu.tr

Ali Göksenli

Tel:+90-2122931300

e-mail: goksenli@itu.edu.tr

Istanbul Technical University

Faculty of Mechanical Engineering

Taksim, Istanbul, Turkey 